\title{
Note on the spectral flow operator
}

\author{
Gaston Giribet \\ Abdus Salam International Centre for Theoretical Physics, ICTP, Strada Costiera 11, Trieste 34151, Italy \\ and Physics Department, University of Buenos Aires and IFIBA-CONICET Ciudad Universitaria, \\ Pabellón 1, Buenos Aires 1428, Argentina
}

(Received 18 July 2019; published 3 December 2019)

\begin{abstract}
The insertion of the spectral flow operator in a string scattering amplitude on $\mathrm{AdS}_{3} \times \mathcal{N}$ produces a change in the winding number of one of the incoming (or outgoing) states, making it possible to compute amplitudes of processes in which the winding number in $\mathrm{AdS}_{3}$ is not conserved. The insertion of such an operator, however, might seem artificial from the world sheet theory perspective, as it appears as an unintegrated vertex operator of conformal dimension zero that does not represent any normalizable state. Here, we show that the spectral flow operator naturally emerges in the Liouville field theory description of the Wess-Zumino-Witten (WZW) correlation functions once it is combined with a series of duality relations among conformal integrals. By considering multiple insertions of spectral flow operators, we study the dependence on the moduli for an arbitrary number of them, and we show explicitly that the amplitude does not depend on the specific locations of the accessory insertions in the world sheet, as required by consistency. This generalizes previous computations in which particular cases were considered. This can also be thought of as an alternative proof of the WZW-Liouville correspondence in the case of maximally winding-violating correlators.
\end{abstract}

DOI: 10.1103/PhysRevD.100.126007

\section{INTRODUCTION}

String theory on $\mathrm{AdS}_{3} \times \mathcal{N}$ with pure Neveu-SchwarzNeveu-Schwarz (NS-NS) fluxes provides an excellent arena to test AdS/CFT correspondence beyond the supergravity approximation [1-3]. The world sheet theory on $\mathrm{AdS}_{3}$, being described by the $S L(2, \mathbb{R})_{k}$ Wess-ZuminoWitten (WZW) model, can be quantized and, in principle, solved exactly. This permits one to have access to the finite $k=R^{2} / \alpha^{\prime}$ regime of the theory, in which the size of the strings, $\ell_{s}=\sqrt{\alpha^{\prime}}$, is not necessarily large in comparison to the radius of the space, $R$. This enables to find the spectrum of the quantum theory in terms of $\widehat{s l}(2)_{k}$ KacMoody unitary representations [4,5] and to compute scattering amplitudes by integrating the WZW correlation functions [6-8].

The results for string amplitudes in $\mathrm{AdS}_{3}$ [9-11], once combined with nonrenormalization theorems that are now available [12], permitted authors in Refs. [13-17] to perform precision checks of AdS/CFT correspondence at large $N$ with $k$ finite. The tree-level three-point functions of

Published by the American Physical Society under the terms of the Creative Commons Attribution 4.0 International license. Further distribution of this work must maintain attribution to the author(s) and the published article's title, journal citation, and DOI. Funded by SCOAP ${ }^{3}$. protected chiral operators in type-IIB superstring theory on $\mathrm{AdS}_{3} \times S^{3} \times T^{4}$ with NS-NS flux were computed in Refs. $[13,14]$ and were shown to exactly reproduce the computations in the symmetric product $\mathrm{CFT}_{2}$ at the orbifold point in the large $N$ limit. The analysis was then extended to the chiral $\mathcal{N}=4$ operators in Ref. [15], and it was later completed in Refs. $[16,17]$ by adding the winding string sectors, which correspond to spectrally flowed representations of $\widehat{s l}(2)_{k}$.

In the past two years, the interest on strings on $\mathrm{AdS}_{3}$ has been renewed. Special attention has recently been focused on the superstring theory at the point $k=1$, where special features appear [18-21]. Different proposals for the holographic description of the supersymmetric theory at $k=1$ have recently been proposed [22-26]. In all such proposals the winding string sectors play a fundamental role. Here, we will study the theory for arbitrary $k$; we will focus on the bosonic theory and analyze the winding string sectors in detail. Our main goal is to clarify the prescription for computing winding-violating string scattering amplitudes proposed in Ref. [6] and make it easy to understand from the world sheet $\mathrm{CFT}_{2}$ point of view.

The paper is organized as follows: In Sec. II, we concisely review string theory on $\mathrm{AdS}_{3}$ with NS-NS fluxes. We study the spectrum and the definition of tree-level scattering amplitudes. Since we will be mainly involved with winding-violating amplitudes, we will need to introduce the so-called spectral flow operator, which will be the 
principal ingredient in our discussion. In Sec. III, we present the most salient properties of the spectral flow operator in relation to correlation functions, and we study the three-point function in detail. In Sec. IV, the main tool to solve the amplitudes is presented: This is the so-called $\mathrm{H}_{3}^{+}$-Liouville correspondence, which is a dictionary that maps $S L(2, \mathbb{R})_{k}$ WZW correlation functions into correlation functions in Liouville field theory (LFT). In Sec. V, we analyze the integral representation of correlation functions in LFT together with a series of duality relations among different conformal integrals that will ultimately lead to compute the relevant three-point functions. In Sec. VI, we comment on the $n$-point functions.

\section{STRING AMPLITUDES ON $\mathrm{AdS}_{3}$}

We will be concerned with tree-level string amplitudes on $\mathrm{AdS}_{3} \times \mathcal{N}$ with pure NS-NS fluxes. These observables are given by

$\mathcal{A}_{\mathbf{p}_{1}, \mathbf{p}_{2}, \ldots \mathbf{p}_{n}}^{n}=\int \prod_{i=1}^{n} d^{2} z_{i} \frac{C_{\mathrm{AdS}_{3}}^{n}\left(z_{1}, \ldots, z_{n}\right) \times C_{\mathcal{N}}^{n}\left(z_{1}, \ldots, z_{n}\right)}{\operatorname{Vol}(\operatorname{PSL}(2, \mathbb{C}))}$,

where the integrals are over $\mathbb{C P}^{1} ; C_{\mathrm{AdS}_{3}}^{n}$ is the $n$-point correlation function of primary operators in the $S L(2, \mathbb{R})_{k}$ WZW model on the Riemann sphere:

$$
C_{\mathrm{AdS}_{3}}^{n}\left(z_{1}, \ldots, z_{n}\right)=\left\langle\prod_{i=1}^{n} \Phi_{j_{i}, m_{i}, \bar{m}_{i}}^{\omega_{i}}\left(z_{i}\right)\right\rangle_{s l(2)}^{\sum_{i=1}^{n} \omega_{i}},
$$

which describes the string $\sigma$ model on $\operatorname{AdS}_{3} ; C_{\mathcal{N}}^{n}$ is the contribution of the internal CFT on $\mathcal{N}$. In Eq. (2), we are omitting normal ordering symbols. Subscript $s l(2)$ on the right-hand side makes explicit that Eq. (2) is a correlator in the noncompact $S L(2, \mathbb{R})$ model. Superscript $\sum_{i=1}^{n} \omega_{i}$ indicates the total winding number in the correlator, as in $\mathrm{AdS}_{3}$ such a quantity is not necessarily conserved [6,7]. The indices $\mathbf{p}_{i}$ in Eq. (1) represent the momenta of the incoming and outgoing states. Both $C_{\mathcal{N}}^{n}$ and $C_{\mathrm{AdS}_{3}}^{n}$ depend on $\mathbf{p}_{i}$, although we do not write explicitly such dependence for short. The volume of the conformal Killing group, $\operatorname{Vol}(\operatorname{PSL}(2, \mathbb{C}))$, can be canceled by fixing on the punctured projective complex plane three of the $n$ points at which the vertices are inserted; as usual, we choose $z_{1}=1-z_{2}=1 / z_{3}=0$.

The spectrum of the theory on $\mathrm{AdS}_{3} \times \mathcal{N}$ was worked out by Maldacena and Ooguri in Ref. [4]. Let us briefly review it here: Operators $\Phi_{j, m, \bar{m}}$ in Eq. (2) represent the $\mathrm{AdS}_{3}$ part of the string vertex operators that create Virasoro primary states in the world sheet CFT. These states organize themselves in representations of the $\widehat{s l}(2)_{k} \oplus \widehat{s l}(2)_{k}$ affine Kac-Moody algebra. These representations are built out of unitary, Hermitian representations of $S L(2, \mathbb{R})$. More precisely, the Hilbert space of the theory is constructed by acting with elements of the enveloping algebra of $\widehat{s l}(2)_{k}$ on states of suitable representations of $S L(2, \mathbb{R})$. Such representations are the highest- and lowest-weight discrete series $\mathcal{D}_{j}^{ \pm}$and the principal continuous series $\mathcal{C}_{j}^{\lambda}$ of $\operatorname{SL}(2, \mathbb{R})$, together with their spectrally flowed images $\mathcal{D}_{j}^{ \pm, \omega}$ and $\mathcal{C}_{j}^{\lambda, \omega}$; see Ref. [4] for details.

The WZW level is given by $k=R^{2} / \alpha^{\prime}$; so it controls the size of the strings relative to the size of $\mathrm{AdS}_{3}$ space, implying that the semiclassical limit corresponds to the limit $k \rightarrow \infty$. The central charge of the world sheet CFT takes the form

$$
c=\frac{3 k}{k-2}+c_{\mathcal{N}} \equiv 26
$$

where $c_{\mathcal{N}}$ is the contribution of the internal CFT on $\mathcal{N}$. This sets the level to be $k=2\left(c_{\mathcal{N}}-26\right) /\left(c_{\mathcal{N}}-23\right)$; as expected, it yields $c_{\mathcal{N}}-26=3$ in the large $k$ limit, in which the three-dimensional AdS space is softly curved.

As said, the indices $\mathbf{p}_{i}$ represent the labels that parameterize the momenta of both the $\mathrm{AdS}_{3}$ and the internal parts; in the $\mathrm{AdS}_{3}$ part these labels are those that classify the $S L(2, \mathbb{R}) \times S L(2, \mathbb{R})$ representations: $j_{i}, m_{i}, \bar{m}_{i}$, and $\omega_{i}$. Discrete representations of the universal covering of $S L(2, \mathbb{R}), \mathcal{D}_{j}^{ \pm}$, correspond to $j \in \mathbb{R}, m \in \pm j \pm \mathbb{Z}_{\geq 0}$, and $\omega=0$; while continuous representations, $\mathcal{C}_{j}^{\lambda}$, correspond to $j \in 1 / 2+i \mathbb{R}, m \in \lambda+\mathbb{Z}$, and $\omega=0$. The sectors $\omega \neq 0$ are defined as the Kac-Moody primaries with respect to the algebra generators obtained by transforming the original ones with a $\mathbb{Z}$-valued spectral flow isomorphism [4], with $\omega \in \mathbb{Z}$ being the spectral flow parameter. The set of unitary, Hermitian representations of $S L(2, \mathbb{R})$ also includes the complementary series $\mathcal{E}_{j}^{\alpha}$, but these series are not necessary to construct the string spectrum. ${ }^{1}$ The spectrum also excludes discrete representations above a certain value $j_{\max }=(k-1) / 2$ in virtue of the strong version of the no-ghost bound [4]. More precisely, one only considers discrete representations in the segment

$$
\frac{1}{2} \leq j \leq \frac{k-1}{2} .
$$

The physical interpretation of the $S L(2, \mathbb{R})$ labels $j, m$, $\bar{m}$, and $\omega$ is the following: The energy of the string states in $\mathrm{AdS}_{3}$ is given by the combination $E \equiv m+\bar{m}+k \omega \in \mathbb{R}$, where $\omega \in \mathbb{Z}$ is the winding number-recall that $k$ is

\footnotetext{
${ }^{1}$ There are two arguments to exclude the complementary series $\mathcal{E}_{j}^{\alpha}$ from the string spectrum. On the one hand, the one-loop partition function of the theory defined only with the series $\mathcal{C}_{j}^{\lambda, \omega}$ and $\mathcal{D}_{j}^{ \pm, \omega}$ results to be modular invariant [27]. On the other hand, the states of the series $\mathcal{C}_{j}^{\lambda, \omega}$ and $\mathcal{D}_{j}^{ \pm, \omega}$ form a basis of the $L^{2}$ functions [4].
} 
proportional to the string tension. The quantity $J \equiv m-$ $\bar{m} \in \mathbb{Z}$ gives the angular momentum in $\mathrm{AdS}_{3}$, and the imaginary part of the variable $j$ can be associated to the radial momentum.

The world sheet conformal dimension of the primary operators on $\mathrm{AdS}_{3} \times \mathcal{N}$ reads $^{2}$

$$
\begin{aligned}
& h=\frac{j(1-j)}{k-2}-m \omega-\frac{k}{4} \omega^{2}+h_{\mathcal{N}}+N, \\
& h=\frac{j(1-j)}{k-2}-\bar{m} \omega-\frac{k}{4} \omega^{2}+\bar{h}_{\mathcal{N}}+\bar{N},
\end{aligned}
$$

where $h_{\mathcal{N}}$ and $\bar{h}_{\mathcal{N}}$ represent the conformal dimensions of the $\sigma$ model on $\mathcal{N}$, and where $N$ and $\bar{N}$ indicate the oscillator numbers. The level-matching condition thus implies $h_{\mathcal{N}}-\bar{h}_{\mathcal{N}} \in \mathbb{Z}$. By imposing the Virasoro constrains on Eq. (5), we obtain the energy spectrum of the theory:

$E=\frac{k}{2} \omega+\frac{1}{\omega}\left(2 \frac{j(1-j)}{k-2}+N_{\mathcal{N}}+\bar{N}_{\mathcal{N}}-2+h_{\mathcal{N}}+\bar{h}_{\mathcal{N}}\right)$.

Spectrally flowed discrete representations $\mathcal{D}_{j \in \mathbb{R}}^{ \pm, \omega}$, which describe short strings confined in the bulk of the space, have a discrete energy spectrum. In contrast, continuous representations $\mathcal{C}_{j \in 1 / 2+i \mathbb{R}}^{\lambda, \omega}$, which describe long strings that can reach the boundary asymptotically with winding number $\omega$, have a continuous energy spectrum.

It is worth mentioning that the winding number in $\mathrm{AdS}_{3}$ is not a topological degree of freedom: $\operatorname{AdS}_{3}$ space is simply connected. Number $\omega$ is rather associated to the presence of the NS-NS $B$ field in the bulk, to which the strings couple. Not being topological, the winding number can in principle change when interactions take placealthough it is preserved in a two-point function. Here, we will be concerned with amplitudes of $n$-string scattering processes in which the total winding number is not conserved. This means that we will compute $S L(2, \mathbb{R})$ WZW correlation functions that include the so-called spectral flow operator [6,7]; namely,

$$
\begin{aligned}
& C_{\mathrm{AdS}_{3}}^{n+1}\left(z_{1}, \ldots, z_{n+1}\right) \\
& \quad=\frac{1}{Z_{\mp}}\left\langle\prod_{i=1}^{n} \Phi_{j_{i}, m_{i}, \bar{m}_{i}}^{\omega_{i}}\left(z_{i}\right) \Phi_{\frac{k}{2}, \pm \frac{k}{2}, \pm \frac{k}{2}}^{\mp 1}\left(z_{n+1}\right)\right\rangle_{s l(2)}^{0} .
\end{aligned}
$$

These correlation functions include one extra operator with fixed momenta $j_{n+1}=k / 2, m_{n+1}=\bar{m}_{n+1}= \pm k / 2$,

\footnotetext{
${ }^{2}$ These formulas are invariant under $j \rightarrow 1-j$ and $m, \bar{m}, \omega \rightarrow$ $-m,-\bar{m},-\omega$. The latter can be regarded as a $C P T$ transformation; states with negative values of $m, \bar{m}$, and $\omega$ have to be regarded as outgoing states.
}

and $\omega_{n+1}=\mp 1$. The superscript 0 on the right-hand side means that $\sum_{i=1}^{n+1} \omega_{i}=\sum_{i=1}^{n} \omega_{i} \mp 1$ gives zero. The $(n+1)$ th operator in Eq. $(7)$ does not represent an external state but is an auxiliary operator. This means that the correct interpretation of a correlator like Eq. (7) is that of an $n$-point function in which the total winding number is violated in one unit, i.e.,

$$
\omega \equiv \sum_{i=1}^{n} \omega_{i}= \pm 1 .
$$

This prescription to compute winding nonpreserving correlators was proposed originally by Fateev, Zamolodchikov, and Zamolodchikov (FZZ) [28]. The idea is that, once integrated in $z_{1}, \ldots, z_{n}$, correlator (7) gives the $n$-point scattering amplitudes $\mathcal{A}_{\mathbf{p}_{1}, \ldots . \mathbf{p}_{n}}^{n}$ of processes that violate the conservation of the total winding number $\omega$ in one unit. This charge condition is induced by the presence of the $(n+1)$ th operator $\Phi_{k / 2, \pm k / 2, \pm k / 2}^{\mp 1}$ inserted at $z_{n+1}$. The FZZ prescription is such that, unlike the other world sheet insertions, $z_{n+1}$ is left unintegrated. This is consistent with the fact that the extra $(n+1)$ th operator has conformal dimension zero.

It is worth emphasizing that operator $\Phi_{k / 2, \mp k / 2, \mp k / 2}^{ \pm 1}$ does not represent any normalizable ${ }^{3}$ state of the theory. In fact, the value $j_{n+1}=k / 2$ violates the unitarity upper bound $j_{\max }=(k-1) / 2$ of the physical spectrum (4). Operator $\Phi_{k / 2, \pm k / 2, \pm k / 2}^{\mp 1}$ is rather an auxiliary tool that is introduced just to alter the total winding number in a given correlator. Such an operator was originally regarded [28] as a "conjugated representations of the identity" operator $\Phi_{0,0,0}^{0}=1$. The relation with the identity operator can be understood algebraically in terms of the identity between states of the representation $\mathcal{D}_{j}^{\mp, 0}$ and states of the representation $\mathcal{D}_{k / 2-j}^{ \pm, \mp 1}$. This is related to the spectral flow isomorphism of $\hat{s l}(2)_{k}$, which for the spectral flow parameter $\omega= \pm 1$ does not necessarily produce new representations ${ }^{4}$ but two different ways of representing the same states. This is the reason why, in Ref. [7], operator $\Phi_{k / 2, \mp k / 2, \mp k / 2}^{ \pm 1}$ was alternatively called "spectral flow operator."

\section{SPECTRAL FLOW OPERATOR}

Let us now discuss the spectral flow operator in a more systematic way: In the WZW theory, we will consider the special dimension-zero local operators

\footnotetext{
${ }^{3}$ One of the properties of the state $j=k / 2=\mp m=\mp \bar{m}$, created by $\Phi_{k / 2, \mp k / 2, \mp k / 2}^{ \pm 1}$, which can be useful to compute correlation functions, is that it contains a null descendant $\lim _{z \rightarrow 0} J_{-1}^{ \pm} \Phi_{k / 2, \mp k / 2, \mp k / 2}^{ \pm 1}(z)|0\rangle=0$.

${ }^{4}$ In particular, operators $\Phi_{0,0,0}^{0}, \Phi_{k / 2, k / 2, k / 2}^{-1}$, and $\Phi_{k / 2,-k / 2,-k / 2}^{1}$ share both the Cartan energy $m+k \omega / 2=0$ and the quadratic Casimir $(k-2) h=0$.
} 


$$
\frac{1}{Z_{+}} \Phi_{\frac{k}{2},-\frac{k}{2},-\frac{k}{2}}^{+1}, \quad \frac{1}{Z_{-}} \Phi_{\frac{k}{2},+\frac{k}{2},+\frac{k}{2}}^{-1},
$$

where the prefactor $Z_{ \pm}^{-1}$ stands for a normalization (possibly divergent) yet to be fixed. This prefactor is somehow related to the $V_{\text {conf }}$ prefactor appearing in Ref. [6].

Being dimension-zero operators with nonvanishing $\omega$, the insertion of Eq. (9) in a correlation function suffices to change the total winding number of a given amplitude without spoiling its conformal properties. Conformal invariance demands the dimension-zero operator not to be integrated. Then, an immediate question that arises is what to do with the inserting points at which they are inserted. This is a question about the structure of the moduli space, and it is one of the questions we want to discuss in detail. In this regard, we will prove that

(a) the final answer for the amplitude does not depend on those insertion points, even when an arbitrary number of spectral flow operators are inserted [this is far from obvious when glancing at Eq. (7), for instance]; and

(b) the final answer for the amplitude only depends on the difference between the number of the operators $\Phi_{k / 2,-k / 2,-k / 2}^{+1}$ and the number of the operators $\Phi_{k / 2,+k / 2,+k / 2}^{-1}$ present in the correlator, regardless of the net number of them.

Showing (a) and (b) requires working out the expressions for the $S L(2, \mathbb{R})_{k}$ WZW correlation functions explicitly, and this is what we will do in the next sections.

The three-point functions are the building block of the higher correlation functions. Therefore, we will focus on the following class of objects:

$$
\begin{aligned}
\tilde{C}_{\mathrm{AdS}_{3}}^{3, n_{+}, n_{-}}= & \frac{1}{Z_{+}^{n_{+}} Z_{-}^{n_{-}}}\left\langle\prod_{i=1}^{3} \Phi_{j_{i}, m_{i}, \bar{m}_{i}}^{\omega_{i}}\left(z_{i}\right) \prod_{a=1}^{n_{+}} \Phi_{\frac{k}{2},-\frac{k}{2},-\frac{k}{2}}^{+1}\left(u_{a}^{+}\right)\right. \\
& \left.\times \prod_{A=1}^{n_{-}} \Phi_{\frac{k}{2},+\frac{k}{2},+\frac{k}{2}}^{-1}\left(u_{A}^{-}\right)\right\rangle_{s l(2)}^{0},
\end{aligned}
$$

which include an arbitrary number $\left(n_{+}+n_{-}\right)$of spectral flow operators. The tilde on $\tilde{C}_{\mathrm{AdS}_{3}}^{3, n_{+}, n_{-}}$is there to remind us of the presence of such operators. World sheet insertion points are fixed at $z_{1}=0, z_{2}=1$, and $z_{3}=\infty$. The superscript 0 on the right-hand side of Eq. (10) indicates that the total winding number vanishes, i.e., $\sum_{i=1}^{3+n_{+}-n_{-}} \omega_{i}=$ $\sum_{i=1}^{3} \omega_{i}+n_{+}-n_{-}=0$. As we discussed, according to the FZZ prescription, correlator (10) actually represents a winding-violating three-point amplitude in which

$$
\sum_{i=1}^{3} \omega_{i}=n_{-}-n_{+}
$$

is generically nonzero. It turns out that these three-point functions vanish if $\left|n_{+}-n_{-}\right|>1$. In fact, one of the remarkable properties of the spectral flow operator is that the violation of the total winding number they induce is bounded: In a tree-level $n$-point scattering amplitude, the total winding number is bounded ${ }^{5}$ by

$$
\left|\sum_{i=1}^{n} \omega_{i}\right| \leq n-2 .
$$

Amplitudes that do not obey this bound vanish identically. This bound, which was originally obtained by direct computation [28], was explained in Ref. [6] in terms of the symmetries of the WZW model. This is also consistent with the Ward identities derived in Ref. [29] and with the bounds obtained in the T-dual model [30].

When considering Eq. (10), two main questions arise:

(i) How to understand the presence of the spectral flow operator in a natural way from the CFT computation point of view. ${ }^{6}$ The way in which we will address this question involves the correspondence between WZW correlators such as Eq. (10) and correlators in LFT. This will lead us to make more precise the analysis of Ref. [31], where the spectral flow operators in relation to Liouville theory was first discussed.

(ii) How to deal with multiple insertions of the spectral flow operator in a given string amplitude. In particular, how to deal with the accessory world sheet marks where the spectral flow operators are inserted. Gauge invariance implies that the amplitude should not depend on those specific points $u_{a}^{ \pm}$. The independence was proven for one or two spectral flow operators $^{7}[6,32]$; here we want to prove this for an arbitrary number of them.

In answering these questions, we will prove statements (a) and (b) above.

\section{IV. $H_{3}^{+}$-LIOUVILLE CORRESPONDENCE REDUX}

To start our proof of (a) and (b), we have to work out the expression for $\tilde{C}_{\mathrm{AdS}_{3}}^{3+n_{+}+n_{-}}$. The first step to compute this correlator is to consider the so-called $\mathrm{H}_{3}^{+}$-Liouville correspondence, which permits us to write $S L(2, \mathbb{R})$ WZW

\footnotetext{
${ }^{5}$ In a genus- $g$ amplitude this bound is expected to be $\left|\sum_{i=1}^{n} \omega_{i}\right| \leq n-2+2 g$.

${ }^{6}$ It was pointed out by Xi Yin that the presence of the spectral flow operator should be derivable from the bootstrap approach in the world sheet $\mathrm{CFT}_{2}$. Here, we will resort to the so-called $\mathrm{H}_{3}^{+}$Liouville correspondence, which follows from the identity between modular differential equations, and therefore this can be seen as an indirect realization of that idea.

${ }^{7}$ Here, we will work in the so-called $m$ basis, in contrast to other works in which the spectral flow operator is written in the Mellin-transformed $x$ basis. In the $m$ basis, the independence from the inserting points $u_{a}^{ \pm}$becomes clearer.
} 
$n$-point correlation functions ${ }^{8}$ in terms of $(2 n-2+\omega)$-point LFT correlation functions, where $\omega$ is the total winding number in the WZW correlator. This correspondence follows from the relation existing between the solutions to the Knizhnik-Zamolodchikov equation and the solutions to the Belavin-Polyakov-Zamolodchikov (BPZ) equation [33]. This was revisited and generalized in Refs. [29,34]. In its general form, which includes the winding number, the $\mathrm{H}_{3}^{+}$-Liouville correspondence formula reads ${ }^{9}$

$$
\begin{aligned}
C_{\mathrm{AdS}_{3}}^{n}\left(z_{1}, \ldots, z_{n}\right)= & \prod_{i=1}^{n} \frac{\Gamma\left(j_{i}-m_{i}\right)}{\Gamma\left(1-j_{i}+\bar{m}_{i}\right)} \sum_{\omega=0}^{n-2} \delta\left(\sum_{i=1}^{n} \omega_{i}-\omega\right) \delta^{(2)}\left(\sum_{i=1}^{n} m_{i}+k \omega / 2\right) \\
& \times \frac{2 \pi^{3-2 n} b\left(c_{b}\right)^{\omega}}{\Gamma(n-1-\omega)} \int^{n-2-\omega} \prod_{a=1}^{2} d^{2} y_{a} \prod_{1 \leq i<i^{\prime}}^{n}\left(\left(z_{i}-z_{i^{\prime}}\right)^{\beta_{i^{\prime}}-2 \kappa_{i} \kappa_{i^{\prime}}}\left(\bar{z}_{i}-\bar{z}_{i^{\prime}}\right)^{\left.\bar{\beta}_{i i^{\prime}}-2 \bar{\kappa}_{i} \bar{\kappa}_{i^{\prime}}\right)}\right. \\
& \times\left\langle\prod_{i=1}^{n} e^{i \sqrt{2}\left(\kappa_{i} \chi\left(z_{i}\right)+\bar{\kappa}_{i} \bar{\chi}\left(\bar{z}_{i}\right)\right)} \prod_{a=1}^{n-2-\omega} e^{i \sqrt{k / 2}\left(\chi\left(y_{a}\right)+\bar{\chi}\left(\bar{y}_{a}\right)\right)}\right\rangle_{\text {free }} \times\left\langle\prod_{i=1}^{n} V_{\alpha_{i}}\left(z_{i}\right) \prod_{a=1}^{n-2-\omega} V_{-\frac{1}{2 b}}\left(y_{a}\right)\right\rangle_{\mathrm{LFT}},
\end{aligned}
$$

where $\quad \kappa_{i}=m_{i} / \sqrt{k}-\sqrt{k} / 2, \quad \bar{\kappa}_{i}=\bar{m}_{i} / \sqrt{k}-\sqrt{k} / 2, \quad \beta_{i i^{\prime}}=\left(1-\omega_{i} \omega_{i^{\prime}}\right) k / 2-\omega_{i} m_{i^{\prime}}-\omega_{i^{\prime}} m_{i}-m_{i}-m_{i^{\prime}}, \quad \bar{\beta}_{i i^{\prime}}=$ $\left(1-\omega_{i} \omega_{i^{\prime}}\right) k / 2-\omega_{i} \bar{m}_{i^{\prime}}-\omega_{i^{\prime}} \bar{m}_{i}-\bar{m}_{i}-\bar{m}_{i^{\prime}}$, and $\delta^{(2)}(z)=\delta(z) \delta(\bar{z}) . c_{b}$ is a $b$-dependent factor that is unimportant here. We are assuming $\sum_{i=1}^{n} \omega_{i} \geq 0$, without loss of generality ${ }^{10}$; an analogous formula exists for $\sum_{i=1}^{n} \omega_{i} \leq 0$. The integrals are defined on the whole $\mathbb{C}$ plane. The first expectation value on the third line of Eq. (13) is a $(2 n-2+\omega)$-point correlation function of primary fields in the theory of a free ${ }^{11}$ boson $\chi(z, \bar{z})=\chi(z)+\bar{\chi}(\bar{z})$. The second expectation value on the third line of Eq. (13) is an $(2 n-2+\omega)$-point function of exponential primary operators in LFT; namely,

$$
\left\langle\prod_{i=1}^{n} V_{\alpha_{i}}\left(z_{i}\right)\right\rangle_{\mathrm{LFT}}=\int \mathcal{D} \varphi e^{-S_{\mathrm{L}}[\varphi]} \prod_{i=1}^{n} e^{\sqrt{2} \alpha_{i} \varphi\left(z_{i}, \bar{z}_{i}\right)}
$$

where the Liouville action is

$$
S_{\mathrm{L}}[\varphi]=\frac{1}{2 \pi} \int d^{2} z\left(\partial \varphi \bar{\partial} \varphi+\frac{Q}{4} R \varphi+\mu e^{\sqrt{2} b \varphi}\right), \quad Q=b+1 / b
$$

The relation between the quantum numbers in the WZW theory and in LFT is

$$
\alpha_{i}=b\left(k / 2-j_{i}\right), \quad b^{2}=1 /(k-2), \quad \mu=b^{2},
$$

with $i=1,2, \ldots, n$. The constant $\mu$ is the Liouville cosmological constant, which here is fixed in terms of $b$. The constant parameter $b$ enters in the LFT central charge as $c=1+6 Q^{2}$. For concreteness, let us consider the case of continuous representations $j_{i}=1 / 2-i p_{i}$ with $p_{i} \in \mathbb{R}$ for $i=1,2,3$, although exactly the same steps hold for arbitrary $\operatorname{SL}(2, \mathbb{R})$ representations. Notice that the relation (16) between the $S L(2, \mathbb{R})$ isospin $j_{i}$ and the LFT momenta $\alpha_{i}$ is such that states of the continuous representation $\mathcal{C}_{j \in 1 / 2+i \mathbb{R}}^{\lambda, \omega}$ get mapped into the normalizable states of LFT, namely, those with $\alpha_{i} \in Q / 2+i \mathbb{R}$.

Out of the $2 n-2-\omega$ operators in the LFT correlator of Eq. (13), $n-2-\omega$ of them have momentum $\alpha=-1 /(2 b)$; see Fig. 1. This special value of the momentum corresponds to non-normalizable, degenerate states in LFT, meaning

\footnotetext{
${ }^{8}$ The $S L(2, \mathbb{R})$ WZW correlation functions $C_{\mathrm{AdS}_{3}}^{n}$ are usually defined by analytic extension from the correlators of the gauged $H_{3}^{+}=S L(2, \mathbb{C}) / S U(2)$ WZW model. The discrete states appear as poles in the amplitudes, while the continuous series follow naturally from the normalizable states of the $H_{3}^{+}$model.

${ }^{9}$ It corresponds to formula (3.29) in Ref. [29] with $r=\omega$ and making $j_{i} \rightarrow-j_{i}$. This is also formula (3.36) of Ref. [34] making $m_{i} \rightarrow-m_{i}, j_{i} \rightarrow-j_{i}$, and setting $\omega_{i}=0$. A generalized version of the formula for arbitrary genus $g$ and $\omega_{i}=0$ was given in Ref. [35], where the $n$-point functions in the $S L(2, \mathbb{R})$ WZW model are in correspondence with the $(2 n-2+2 g)$-point functions of LFT.

${ }^{10}$ To be more precise, the step $\omega=n-2$ of formula (13) was not proven in Ref. [29] but it was presented as an educated conjecture, the reason being that in that case there are no degenerate fields in the LFT correlator and so its form cannot be constrained by the BPZ

equation. The case $\omega=n-2$ was later proven in Ref. [36] using free field techniques.
${ }^{11}$ It can also be realized as an $n$-point correlation function of a $c<1$ matter CFT coupled to LFT, where the operators $e^{i \sqrt{k / 2}} \chi \times$ $V_{-1 /(2 b)}$ act as screening charges; this also explains the factor $c_{b}^{\omega} / \Gamma(n-1-\omega)$ as coming from combinatorics.
} 

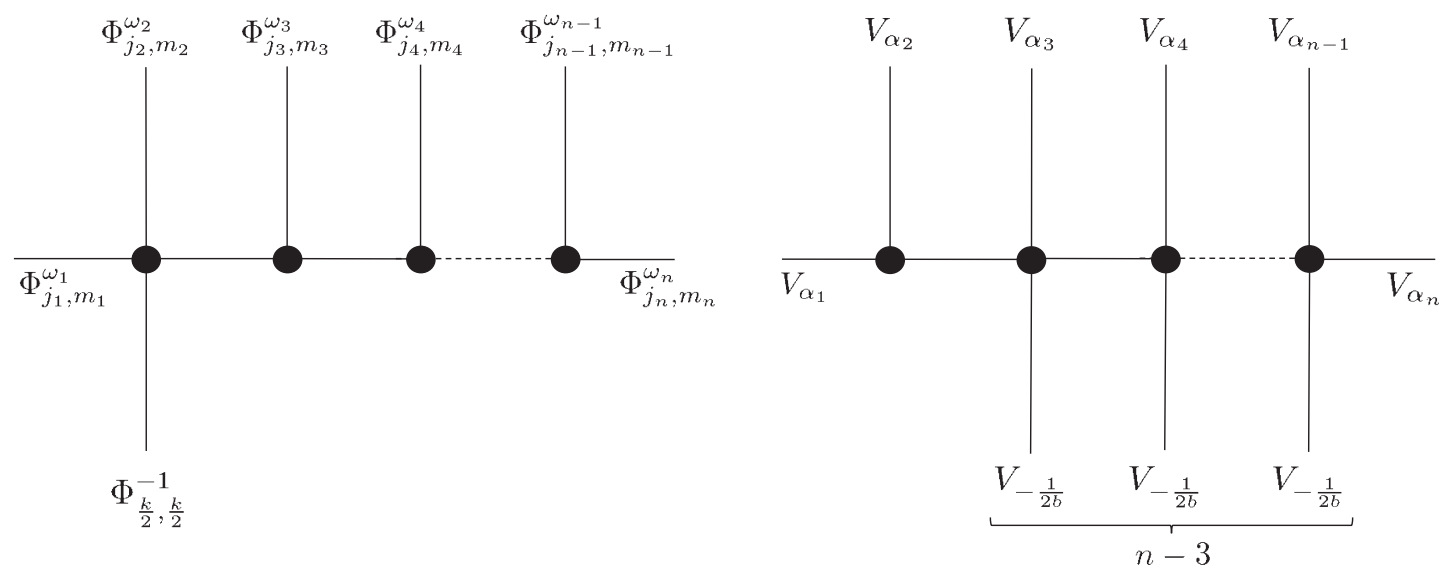

FIG. 1. Scheme of the $H_{3}^{+}$-Liouville correspondence. On the left, an $n$-point string scattering amplitude in AdS $S_{3}$ in which the total winding number is $\omega \equiv \omega_{1}+\omega_{2}+\cdots+\omega_{n}=1$; it is defined as an $(n+1)$-point correlation function in the $S L(2 \text {, } \mathbb{R})_{k}$ WZW model involving one (as $\omega=1)$ spectral flow operator. On the right, the corresponding $(2 n-3)$-point correlation function in LFT involving $n-2-\omega$ degenerate fields $V_{-1 /(2 b)}$ integrated on the sphere.

that $n-2-\omega$ states in the LFT correlator contain null descendants in the Verma modulo. The number of degenerate fields $V_{-1 /(2 b)}$ controls the violation of the winding number. This number goes from $0(\omega=n-2)$ to $n-2(\omega=0)$. In this formula, the bound $\omega \leq n-2$ comes from the Ward identities (2.31) of Ref. [29], whose solution is the product of $\omega+1 \delta$ functions in Eq. (3.26) therein. ${ }^{12}$

We can use the $H_{3}^{+}$-Liouville correspondence formula (13) to write the correlator (10) as

$$
\begin{aligned}
\tilde{C}_{\mathrm{AdS}_{3}}^{3, n_{+}, n_{-}}= & \lim _{\epsilon \rightarrow 0} \frac{(\Gamma(\epsilon))^{n_{-}}}{Z_{+, \epsilon}^{n_{+}} Z_{-, \epsilon}^{n_{-}}} \frac{2(\Gamma(k))^{n_{+}} \pi^{-3-2 n_{+}-2 n_{-}}}{\sqrt{k-2}(\Gamma(1-k))^{n_{+}} \Gamma\left(2+n_{+}+n_{-}\right)} \prod_{a=1}^{3} \frac{\Gamma\left(\frac{1}{2}-i p_{a}-m_{a}\right)}{\Gamma\left(\frac{1}{2}+i p_{a}+m_{a}\right)} \\
& \times \delta^{(2)}\left(\sum_{a=1}^{3} m_{a}-\frac{k}{2}\left(n_{+}-n_{-}\right)\right) \prod_{a=1}^{n_{+}}\left(\left|u_{a}^{+}\right|^{2 k-4 m_{1}}\left|1-u_{a}^{+}\right|^{2 k-4 m_{2}}\right) \\
& \times \prod_{1 \leq a<a^{\prime}}^{n_{+}}\left|u_{a}^{+}-u_{a^{\prime}}^{+}\right|^{4 k} \int^{n_{+}} \prod_{l=1}^{+n_{-}+1} d^{2} y_{l} \prod_{1 \leq l<l^{\prime}}^{n_{+}+n_{-}+1}\left|y_{l}-y_{l^{\prime}}\right|^{k} \prod_{a=1}^{n_{+}} \prod_{l=1}^{n_{+}+n_{-}+1}\left|u_{a}^{+}-y_{l}\right|^{-2 k} \\
& \times \prod_{l=1}^{n_{+}+n_{-}+1}\left(\left|y_{l}\right|^{2 m_{1}-k}\left|1-y_{l}\right|^{2 m_{2}-k}\right)\left\langle V_{\alpha_{1}}(0) V_{\alpha_{2}}(1) V_{\alpha_{3}}(\infty) \prod_{l=1}^{n_{+}+n_{-}+1} V_{-\frac{1}{2 b}}\left(y_{l}\right)\right\rangle_{\mathrm{LFT}},
\end{aligned}
$$

where $\alpha_{i}=b\left(-j_{i}+b^{-2} / 2+1\right)=\left(k / 2-1 / 2+i p_{i}\right) / \sqrt{k-2}$, $i=1, \quad 2, \quad 3, \quad$ with $j_{i}=1 / 2-i p_{i}, \quad p_{i} \in \mathbb{R}, \quad$ and $b=1 / \sqrt{k-2}$. This implies that for the remaining operators we have $\alpha_{a>3}=0$ with $a=4,5, \ldots, n_{-}+n_{+}$.

We already see some magic working here: All the dependence on the variables $u_{A}^{-}$has disappeared from the expression above. We will see next that, by rewriting this expression in a smart way, also the remaining variables

\footnotetext{
${ }^{12}$ Using the notation of Ref. [29], if $\omega \geq n-1$, one finds at least $n$ delta functions for $n$ variables $\mu_{i}$, which together impose $\mu_{i}=0$; i.e., the $n$-point function with $\omega \geq n-1$ vanishes almost everywhere in $\mu$ space, in agreement with inequality (12) herein. The author thanks Sylvain Ribault for clarifying this point.
}

$u_{a}^{+}$are seen to be superfluous. This is not evident from Eq. (17), but it will become clear after some algebraic manipulation.

In the expression above, the subscript $\epsilon$ in the normalization factors $Z_{ \pm, \epsilon}$ appears because we want to use the freedom in the normalization of the spectral flow operators to eventually cancel the divergent factor $(\Gamma(\epsilon \rightarrow 0))^{n_{-}}$ together with other divergences that might eventually appear through the calculation.

From Eq. (10), we have $\sum_{i=1}^{3} \omega_{i}+n_{+}-n_{-}=0$. We will consider the case $n_{+}-n_{-}=-1$, which corresponds to $\sum_{i=1}^{3} \omega_{i}=1$. It involves the contribution of the $n_{+}+n_{-}=2 n_{-}-1$ spectral flow operators, with $n_{-}$ being arbitrary. In other words, it corresponds to 
$\sum_{i=1}^{3} m_{i}=\sum_{i=1}^{3} \bar{m}_{i}=\left(n_{+}-n_{-}\right) k / 2=-k / 2$. We also assume $m_{i}=\bar{m}_{i}$ for simplicity.

Our strategy will be the following: (i) We will start with the expression (17) for $\tilde{C}_{\mathrm{AdS}_{3}}^{3+n_{-}+n_{+}}$, in which we used the step $\omega=0$ of the formula (13) to express $\tilde{C}_{\mathrm{AdS}_{3}}^{3+n_{+}+n_{+}}$in terms of a LFT correlator. (ii) We will work out the expression as much as we can using a series of integral identities and duality relations. (iii) We will resort again to formula (13) and use the step $\omega=1$ of the sum to verify that, when $n_{-}=n_{+}+1$, the original quantity $\tilde{C}_{\mathrm{AdS}_{3}}^{3+n_{-}+n_{+}}$actually coincides with the $S L(2, \mathbb{R})$ WZW three-point amplitude that violates the winding number in +1 . In other words, we will see that the step $\omega=0$ of the sum appearing in formula
(13), when $n_{-}-n_{+}=1$ spectral flow operators are considered, coincides with the step $\omega=1$ of the same formula (13) when no spectral flow operators are present. That is to say, we will prove the consistency between the FZZ prescription and the $\mathrm{H}_{3}^{+}$-Liouville correspondence; see Eq. (25) below.

\section{INTEGRAL REPRESENTATION AND DUALITY}

In order to get a closed expression for $\tilde{C}_{\mathrm{AdS}_{3}}^{3, n_{+}, n_{-}}$, we still need to solve the LFT correlators on the right-hand side of Eq. (17). To do so, we could try with the standard Coulomb gas realization [37], namely,

$$
\left\langle\prod_{i=1}^{\tilde{n}} V_{\alpha_{i}}\left(z_{i}\right)\right\rangle_{\mathrm{LFT}}=\mu^{\tilde{s}} \Gamma(-\tilde{s}) \prod_{1 \leq i<i^{\prime}}^{\tilde{n}}\left|z_{i}-z_{i^{\prime}}\right|^{-4 \alpha_{i} \alpha_{i^{\prime}}} I_{\tilde{s}}\left(\alpha_{1}, \ldots, \alpha_{\tilde{n}} ; z_{1}, \ldots, z_{\tilde{n}} ; b\right)
$$

with

$$
I_{\tilde{s}}\left(\alpha_{1}, \ldots, \alpha_{\tilde{n}} ; z_{1}, \ldots, z_{\tilde{n}} ; b\right)=\int \prod_{r=1}^{\tilde{s}} d^{2} w_{r} \prod_{i=1}^{\tilde{n}} \prod_{r=1}^{\tilde{s}}\left|z_{i}-w_{r}\right|^{-4 b \alpha_{i}} \prod_{1 \leq r<r^{\prime}}^{\tilde{s}}\left|w_{r}-w_{r^{\prime}}\right|^{-4 b^{2}}
$$

where $\tilde{s}=1+b^{-2}-b^{-1} \sum_{i=1}^{\tilde{n}} \alpha_{i}$ and where the integrals are on the whole $\mathbb{C}$ plane. The factor $\mu^{\tilde{s}} \Gamma(-\tilde{s})$ in Eq. (18), together with the $\tilde{s}$ insertions at $w_{1}, w_{2}, \ldots, w_{\tilde{s}}$, comes from the integration over the zero mode of the Liouville field $\langle\varphi\rangle=\varphi-\delta \varphi$, and $\mu$ is the Liouville cosmological constant. However, although realization (18) turns out to be useful in many contexts, it would not be of help here. Instead, it is convenient to resort to its dual realization, which amounts to use the self-duality that LFT exhibits under $b \leftrightarrow b^{-1}$. This permits us to alternatively express the LFT correlators as follows:

$$
\left\langle\prod_{i=1}^{\tilde{n}} V_{\alpha_{i}}\left(z_{i}\right)\right\rangle_{\mathrm{LFT}}=\tilde{\mu}^{s} \Gamma(-s) \prod_{1 \leq i<i^{\prime}}^{\tilde{n}}\left|z_{i}-z_{i^{\prime}}\right|^{-4 \alpha_{i} \alpha_{i^{\prime}}} I_{s}\left(\alpha_{1}, \ldots, \alpha_{\tilde{n}} ; z_{1}, \ldots, z_{\tilde{n}} ; 1 / b\right),
$$

where $s=b^{2} \tilde{s} \quad$ and $\quad \tilde{\mu}=\mu^{-b^{-2}} \pi^{b^{-2}-1} \Gamma\left(1-b^{-2}\right)$ $\left(\Gamma\left(b^{2}\right)\right)^{b^{-2}}\left(\Gamma\left(1-b^{2}\right)\right)^{-b^{-2}}\left(\Gamma\left(b^{-2}\right)\right)^{-1}$. Here, because of Eq. (16), we have a particular value of $\mu$ and therefore of $\tilde{\mu}$. Nevertheless, we prefer to write the expressions for generic $\tilde{\mu}$ as it permits to keep track of the Knizhnik-Polyakov-Zamolodchikov scaling of the correlators and to have control over the string coupling constant on $\mathrm{AdS}_{3}$ [38].

The ones appearing on the right-hand side of Eq. (20) are $s$ Selberg-type integrals over $C P^{1} \backslash\left\{z_{1}, \ldots, z_{n}\right\}$. The measure is $d^{2} w_{r}=(i / 2) d w_{r} d \bar{w}_{r}$ with, say, $w_{r}=x_{r}+i y_{r}$ and $\bar{w}_{r}=x_{r}-i y_{r}$. To solve Eq. (20) it is convenient to Wick rotate $x_{r} \rightarrow i x_{r}$ and introduce a deformation parameter in $\left|w_{r}\right|^{2}=-x_{r}^{2}+y_{r}^{2}+i \varepsilon$ to avoid the poles at $x_{r}= \pm y_{r}$. The way to proceed is first to define coordinates $x_{r}^{ \pm}= \pm x_{r}+y_{r}$ and then integrate over $x_{r}^{-}$while keeping $x_{r}^{+}$fixed. ${ }^{13}$

Of course, the integral formula (18) only makes sense for kinematical configurations such that $\tilde{s} \in \mathbb{Z}_{\geq 0}$. This is because $s$ in Eq. (19) is the number of integrals to be performed. Nevertheless, one can make sense out of Eq. (20) in more general cases: To do so, one first assumes $s \in \mathbb{Z}_{\geq 0}$, then solves the integrals in terms of elementary functions, and finally analytically continues the expressions to $s \in \mathbb{C}$. Here, throughout the formulas, we will assume this kind of analytic extension.

\footnotetext{
${ }^{13}$ The author thanks Matías Leoni for discussions about this point.
} 


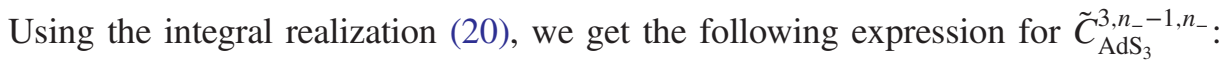

$$
\begin{aligned}
\tilde{C}_{\mathrm{AdS}_{3}}^{3, n_{-}-1, n_{-}}= & \lim _{\epsilon \rightarrow 0} \frac{(\Gamma(\epsilon))^{n_{-}}}{Z_{+, \epsilon}^{n_{-}-1} Z_{-, \epsilon}^{n_{-}}} \frac{2 \Gamma(-s)(\Gamma(k))^{n_{-}-1} \tilde{\mu}^{s} \pi^{-4 n_{-}-1}}{\sqrt{k-2}(\Gamma(1-k))^{n_{-}-1} \Gamma\left(2 n_{-}+1\right)} \prod_{a=1}^{3} \frac{\Gamma\left(\frac{1}{2}-i p_{a}-m_{a}\right)}{\Gamma\left(\frac{1}{2}+i p_{a}+m_{a}\right)} \\
& \times \delta^{(2)}\left(\sum_{a=1}^{3} m_{a}+\frac{k}{2}\right) \int \prod_{l=1}^{2 n_{-}} d^{2} y_{l} \int \prod_{r=1}^{s} d^{2} w_{r} \prod_{1 \leq l<l^{\prime}}^{2 n_{-}}\left|y_{l}-y_{l^{\prime}}\right|^{2} \prod_{1 \leq r<r^{\prime}}^{s}\left|w_{r}-w_{r^{\prime}}\right|^{8-4 k} \\
& \times \prod_{l=1}^{2 n_{-}} \prod_{r=1}^{s}\left|y_{l}-w_{r}\right|^{2 k-4} \prod_{1 \leq a<a^{\prime}}^{n_{-}-1}\left|u_{a}^{+}-u_{a^{\prime}}^{+}\right| 4 k \prod_{a=1}^{n_{-}-1} \prod_{l=1}^{2 n_{-}}\left|u_{a}^{+}-y_{l}\right|^{-2 k} \prod_{a=1}^{n_{-}-1}\left(\left|u_{a}^{+}\right|^{2 k-4 m_{1}}\left|1-u_{a}^{+}\right|^{2 k-4 m_{2}}\right) \\
& \times \prod_{l=1}^{2 n_{-}}\left(\left|y_{l}\right|^{2 m_{1}-1+2 i p_{1}}\left|1-y_{l}\right|^{2 m_{2}-1+2 i p_{2}}\right) \prod_{r=1}^{s}\left(\left|w_{r}\right|^{2-4 i p_{1}-2 k}\left|1-w_{r}\right|^{2-4 i p_{2}-2 k}\right)
\end{aligned}
$$

with $s(k-2)=-\sum_{i=1}^{3} \alpha_{i} / b+\left(1+n_{+}+n_{-}\right) /\left(2 b^{2}\right)+1+1 / b^{2}=-i \sum_{i=1}^{3} p_{i}-1 / 2+(k / 2-1)\left(2 n_{-}-1\right)$. Notice the factor $(\Gamma(1-k))^{1-n_{-}}$in the expression above, which for $k \in \mathbb{Z}_{>2}$ represents a factor zero for $n_{-}>1$. This factor can be eventually absorbed in the normalization $\left(Z_{+, \epsilon}\right)^{1-n_{-}}$, together with other factors; see below. The factor $\Gamma(-s)$, which is divergent for $s \in \mathbb{Z}_{\geq 0}$, is the well-known factor that accompanies the resonant correlators in the Coulomb gas realization; this leads to the residues of the observables with the appropriate factor once one uses the relation $\Gamma(-s) \sim \Gamma(0)(-1)^{s} / s$ ! and isolates the single pole.

Expression (21) is cumbersome and somehow obscure: In particular, it does not make explicit the fact that $\tilde{C}_{\mathrm{AdS}_{3}}^{3, n_{-}-1, n_{-}}$does not depend on the variables $u_{a}^{+}$. Therefore, it is still necessary to simplify it further. To do so, we can resort to the integral relation $^{14}[39,40]$

$$
\begin{aligned}
& \prod_{i=1}^{n} d^{2} y_{i} \prod_{1 \leq 1<i^{\prime}}^{n}\left|y_{i}-y_{i^{\prime}}\right|^{2} \prod_{i=1}^{n} \prod_{i=1}^{n+m+1}\left|y_{i}-x_{j}\right|^{2 L_{j}} \\
& =\pi^{n-m} \frac{\Gamma(n+1)}{\Gamma(m+1)} \frac{\Gamma\left(-n-\sum_{j=1}^{n+m+1} L_{j}\right)}{\Gamma\left(1+n+\sum_{j=1}^{n+m+1} L_{j}\right)} \prod_{j=1}^{n+m+1} \frac{\Gamma\left(1+L_{j}\right)}{\Gamma\left(-L_{j}\right)} \prod_{1 \leq j<j^{\prime}}^{n+m+1}\left|x_{j}-x_{j^{\prime}}\right|^{2+2 L_{j}+2 L_{j^{\prime}}} \\
& \quad \times \prod_{l=1}^{m} d^{2} y_{l} \prod_{1 \leq l<l^{\prime}}^{m}\left|y_{l}-y_{l^{\prime}}\right|^{2} \prod_{l=1}^{m} \prod_{j=1}^{n+m+1}\left|y_{l}-x_{j}\right|^{-2-2 L_{j}} .
\end{aligned}
$$

We can use this formula to change the number of integrals over the variables $y_{l}$ from $n_{+}+n_{-}+1=2 n_{-}$to $s-n_{-}$. This amounts to considering in Eq. (22) the case $n=2 n_{-}, m=s-n_{-}$. This yields

$$
\begin{aligned}
\tilde{C}_{\mathrm{AdS}_{3}}^{3, n_{-}-1, n_{-}}= & -\lim _{\epsilon \rightarrow 0} \frac{(\Gamma(\epsilon))^{n_{-}}}{Z_{+, \epsilon}^{n_{-}-1} Z_{-, \epsilon}^{n_{-}}} \frac{2 \Gamma(-s) \pi^{-1-n_{-}-s}}{\sqrt{k-2} \Gamma\left(s-n_{-}+1\right)}\left(\tilde{\mu} \frac{\Gamma(k-1)}{\Gamma(2-k)}\right)^{s} \\
& \times \delta^{(2)}\left(\sum_{a=1}^{3} m_{a}+\frac{k}{2}\right) \int \prod_{l=1}^{s-n_{-}} d^{2} y_{l} \int \prod_{r=1}^{s} d^{2} w_{r} \prod_{1 \leq l<l^{\prime}}^{s-n_{-}}\left|y_{l}-y_{l^{\prime}}\right|^{2} \prod_{1 \leq r<r^{\prime}}^{s}\left|w_{r}-w_{r^{\prime}}\right|^{2} \\
& \times \prod_{l=1}^{s-n_{-}} \prod_{r=1}^{s}\left|y_{l}-w_{r}\right|^{2-2 k} \prod_{r=1}^{s} \prod_{a=1}^{n_{-}-1}\left|w_{r}-u_{a}^{+}\right|^{-2} \prod_{1 \leq a<a^{\prime}}^{n_{-}-1}\left|u_{a}^{+}-u_{a^{\prime}}^{+}\right|^{n_{-}-1} \prod_{a=1}^{s-n_{-}} \prod_{l=1}^{s-u_{a}^{+}-\left.y_{l}\right|^{2 k-2}} \\
& \times \prod_{a=1}^{n_{-}-1}\left(\left|u_{a}^{+}\right|^{1+2 i p_{1}-2 m_{1}}\left|1-u_{a}^{+}\right|^{1+2 i p_{2}-2 m_{2}}\right) \prod_{l=1}^{s-n}\left(\left|y_{l}\right|^{-1-2 i p_{1}-2 m_{1}}\left|1-y_{l}\right|^{-1-2 i p_{2}-2 m_{2}}\right) \\
& \times \prod_{r=1}^{s}\left(\left|w_{r}\right|^{-1+2 m_{1}-2 i p_{1}}\left|1-w_{r}\right|^{-1+2 m_{2}-2 i p_{2}}\right) .
\end{aligned}
$$

\footnotetext{
${ }^{14}$ As a consistency check, we can use integral formula (22) together with the $H_{3}^{+}$-Liouville correspondence formula (13) to write the resonant WZW $n$-point correlators with momenta satisfying $\sum_{i=1}^{n} j_{i}=n-1$ and $\sum_{i=1}^{n} \omega_{i}=0$ and show they take the form $C_{\mathrm{AdS}}^{n} \propto$ $\prod_{1 \leq i<j}^{n}\left|z_{i}-z_{j}\right|^{-4 t_{i j}}$ with $t_{i j}=\left(1-j_{i}\right)\left(1-j_{j}\right) /(k-2)+\left(m_{i}+k \omega_{i} / 2\right)\left(m_{i}+k \omega_{i} / 2\right)-m_{i} m_{j}$. This expression can be derived independently, for example using the Wakimoto free field representation.
} 
This expression, although still complicated, exhibits some promising simplifications with respect to Eq. (21). In particular, we notice that the exponent 2 in the factors $\left|w_{r}-w_{r^{\prime}}\right|^{2}$ permits one to use formula (22) again to integrate out the variables $w_{r}$. Considering in Eq. (22) the case $n=s, m=0$, the expression for $\tilde{C}_{\mathrm{AdS}_{3}}^{3, n_{-}-1, n_{-}}$reduces to

$$
\begin{aligned}
\tilde{C}_{\mathrm{AdS}_{3}}^{3, n_{-}-1, n_{-}}= & \lim _{\epsilon \rightarrow 0} \frac{(\Gamma(\epsilon))^{2 n_{-}-1}}{Z_{+, \epsilon}^{n_{-}-1} Z_{-, \epsilon}^{n_{-}}}\left(-\frac{\tilde{\mu}}{\pi} \frac{\Gamma(k-1)}{\Gamma(2-k)}\right)^{n_{-}} \prod_{a=1}^{3} \frac{\Gamma\left(\frac{1}{2}-i p_{a}+m_{a}\right)}{\Gamma\left(\frac{1}{2}+i p_{a}-m_{a}\right)} \delta^{(2)}\left(\sum_{a=1}^{3} m_{a}+\frac{k}{2}\right) \\
& \times \frac{2 b c_{b}}{\pi^{3}} \tilde{\mu}^{s-n_{-}} \Gamma\left(n_{-}-s\right) \prod_{l=1}^{s-n_{-}} d^{2} y_{l} \prod_{1 \leq l<l^{\prime}}^{s-n_{-}}\left|y_{l}-y_{l^{\prime}}\right|^{8-4 k} \prod_{l=1}^{s-n_{-}}\left(\left|y_{l}\right|^{4 i p_{1}+2-2 k}\left|1-y_{l}\right|^{4 i p_{2}+2-2 k}\right),
\end{aligned}
$$

where we have used that $\Gamma(-s) \Gamma(s+1)=(-1)^{n_{-}}$ $\Gamma\left(s-n_{-}+1\right) \Gamma\left(n_{-}-s\right) . c_{b}$ stands for an irrelevant factor, which depends on $b$ but does not depend either on the momenta $p_{i}$ or on $m_{i}$.

Remarkably, as we anticipated, the variables $u_{a}^{+}$have totally disappeared from the expression. Moreover, using Eq. (20) we can recognize the second line of the last expression as the integral representation of a LFT threepoint function. This yields

$$
\begin{aligned}
\tilde{C}_{\mathrm{AdS}_{3}}^{3, n_{+}, n_{-}}= & \frac{2 b c_{b}}{\pi^{3}} \prod_{a=1}^{3} \frac{\Gamma\left(\frac{1}{2}-i p_{a}+m_{a}\right)}{\Gamma\left(\frac{1}{2}+i p_{a}-m_{a}\right)} \delta^{(2)}\left(\sum_{a=1}^{3} m_{a}+\frac{k}{2}\right) \\
& \times\left\langle\prod_{i=1}^{3} V_{\alpha_{i}}\left(z_{i}\right)\right\rangle_{\mathrm{LFT}}
\end{aligned}
$$

where $\alpha_{i}=b\left(k / 2-1 / 2-i p_{i}\right)$. We have absorbed in the normalization some factors; more precisely, we fixed $Z_{+, \epsilon}=\Gamma(\epsilon)$ and $Z_{-, \epsilon}=Z_{+, \epsilon} \tilde{\mu} \Gamma\left(b^{-2}\right) /\left(b^{4} \pi \Gamma\left(1-b^{-2}\right)\right)-$ this can actually be seen as a consistency check, since it was not a priori obvious that both the dependence on the arbitrary number $n_{-}$and the divergences could have been absorbed in the normalization factors.

We can express Eq. (24) in terms of the $\Upsilon$ functions introduced in Ref. [41] by simply evaluating the DornOtto-Zamolodchikov-Zamolodchikov formula for the LFT three-point function. Besides, resorting to the functional identity ${ }^{15} G(x)=b^{-b^{2} x^{2}-\left(b^{2}+1\right) x} \Upsilon_{b}^{-1}(-b x)$, we can easily show that expression (24) reproduces the results of Refs. $[6,7,36,42]$ for the winding-violating three-point amplitude in $\mathrm{AdS}_{3}$.

\footnotetext{
${ }^{15}$ This function is defined in terms of the Barnes double Gamma function, $\Gamma_{2}(a \mid b, c)$, as follows: $G(j)=\Gamma_{2}(-j \mid 1, k-2)$ $\Gamma_{2}(k-1+j \mid 1, k-2)(k-2)^{j(k-1-j) /(2 k-4)}$. See (2.13)-(2.16) in Ref. [6] and references therein.
}

Alternatively, we can revert the argument and use again formula (13) to verify that the right-hand side of Eq. (24) is nothing but the winding-violating WZW three-point function itself. Then, we conclude

$$
\begin{gathered}
\left\langle\prod_{i=1}^{3} \Phi_{\frac{1}{2}-p_{i}, m_{i}, \bar{m}_{i}}^{\omega_{i}} \prod_{a=1}^{n_{-}-1} \Phi_{\frac{k}{2},-\frac{k}{2},-\frac{k}{2}}^{+1} \prod_{A=1}^{n_{-}} \Phi_{\frac{k}{2},+\frac{k}{2},+\frac{k}{2}}^{-1}\right\rangle_{s l(2)}^{0} \\
=Z_{+}^{n_{-}-1} Z_{-}^{n_{-}}\left\langle\prod_{i=1}^{3} \Phi_{\frac{1}{2}+p_{i},-m_{i},-\bar{m}_{i}}^{\omega_{i}}\right\rangle_{s l(2)}^{1}
\end{gathered}
$$

This is precisely the identity we wanted to prove: The $\left(2+2 n_{-}\right)$-point function $\tilde{C}_{\mathrm{AdS}_{3}}^{3, n_{-}-1, n_{-}}$actually gives the three-point function $C_{\mathrm{AdS}_{3}}^{3}$ with total winding number $\sum_{i=1}^{3} \omega_{i}=1$.

\section{THE $\boldsymbol{n}$-POINT FUNCTION}

Expression (24) and (25) manifestly show the independence from the inserting points of the auxiliary spectral flow operators, i.e., $\partial_{u_{a}^{ \pm}} \tilde{C}_{\mathrm{AdS}_{3}}^{3, n_{-}-1, n_{-}}=0$. This is far from evident if one starts, for example, with representation (21), which seemed to depend at least on the $u_{a}^{+}$. Furthermore, we argued that, after having chosen the adequate normalization, the result does not depend on $n_{-}$and $n_{+}$separately, but it depends on the difference $n_{-}-n_{+}$. In other words, the vertices $\Phi_{k / 2, k / 2, k / 2}^{-1}$ and $\Phi_{k / 2,-k / 2,-k / 2}^{1}$ neutralize each other.

Going back to the independence from $u_{a}^{ \pm}$, we notice that a similar phenomenon happens with $n$-point functions: Consider for example the $n$-point function

$$
\tilde{C}_{\mathrm{AdS}_{3}}^{n, 0, n_{-}}\left(z_{1}, \ldots, z_{n}\right)=\left\langle\prod_{i=1}^{n} \Phi_{j_{i}, m_{i}, \bar{m}_{i}}^{\omega_{i}}\left(z_{i}\right) \prod_{a=1}^{n_{-}} \Phi_{\frac{k}{2},+\frac{k}{2},+\frac{k}{2}}^{-1}\left(u_{a}^{-}\right)\right\rangle_{s l(2)}^{0} .
$$


Using Eqs. (13) and (20), this yields

$$
\begin{aligned}
\tilde{C}_{\mathrm{AdS}_{3}}^{n, 0, n_{-}}\left(z_{1}, \ldots, z_{n}\right)= & \lim _{\epsilon \rightarrow 0} \frac{(\Gamma(\epsilon))^{n_{-}}}{Z_{-, \epsilon}^{n_{-}}} \frac{2 \Gamma(-s) \tilde{\mu}^{s} \pi^{-2 n_{-}-3}}{\sqrt{k-2} \Gamma\left(n-1+n_{-}\right)} \prod_{1 \leq i<j}^{n}\left|z_{i}-z_{j}\right|^{2 \hat{\beta}_{i j}} \prod_{a=1}^{n} \frac{\Gamma\left(\frac{1}{2}-i p_{a}-m_{a}\right)}{\Gamma\left(\frac{1}{2}+i p_{a}+m_{a}\right)} \\
& \times \delta^{(2)}\left(\sum_{a=1}^{3} m_{a}+\frac{k}{2} n_{-}\right) \int \prod_{l=1}^{n_{-}+n-2} d^{2} y_{l} \int \prod_{r=1}^{s} d^{2} w_{r} \prod_{1 \leq l<l^{\prime}}^{n_{-}+n-2}\left|y_{l}-y_{l^{\prime}}\right|^{2} \prod_{1 \leq r<r^{\prime}}^{s}\left|w_{r}-w_{r^{\prime}}\right|^{8-4 k} \\
& \times \prod_{l=1}^{n_{-}+n-2} \prod_{r=1}^{s}\left|y_{l}-w_{r}\right|^{2 k-4} \prod_{a=1}^{n} \prod_{l=1}^{n_{-}+n-2}\left|z_{a}-y_{l}\right|^{2 m_{a}-1+2 i p_{a}} \prod_{b=1}^{n} \prod_{r=1}^{s}\left|z_{b}-w_{r}\right|^{2-4 i p_{b}-2 k}
\end{aligned}
$$

with $s(k-2)=-i \sum_{i=1}^{n} p_{i}+1-n / 2+(k / 2-1) n_{-}$and $\hat{\beta}_{i j}=\beta_{i j}-2 \alpha_{i} \alpha_{j}$. This expression is actually independent from $u_{a}^{-}$, although it does depend on $n_{-}$yielding $\sum_{i=1}^{n} \omega_{i}=n_{-}$. The independence from the variables $u_{a}^{-}$is here observed at the level of $n$-point correlation functions and not only at the level of correlation numbers or amplitudes.

\section{ACKNOWLEDGMENTS}

The author is indebted to Matthew Kleban and Massimo Porrati for many discussions and ideas. He is also grateful to Christopher Hull, Matías Leoni, Yu Nakayama, and Eliezer Rabinovici for enjoyable collaborations in related subjects. He has also been benefited by discussions with Sylvain Ribault and Xi Yin. Finally, he thanks the hospitality of the following institutions, where different parts of this work were done: the Abdus Salam International Centre for Theoretical Physics, the Center for Cosmology and Particle Physics of New York University, and the Simons Center for Geometry and Physics at Stony Brook University.
[1] A. Giveon, D. Kutasov, and N. Seiberg, Comments on string theory on $\operatorname{AdS}(3)$, Adv. Theor. Math. Phys. 2, 733 (1998).

[2] D. Kutasov and N. Seiberg, More comments on string theory on AdS(3), J. High Energy Phys. 04 (1999) 008.

[3] J. de Boer, H. Ooguri, H. Robins, and J. Tannenhauser, String theory on AdS(3), J. High Energy Phys. 12 (1998) 026.

[4] J. M. Maldacena and H. Ooguri, Strings in AdS(3) and SL $(2, \mathrm{R})$ WZW model 1: The spectrum, J. Math. Phys. (N.Y.) 42, 2929 (2001).

[5] G. Giribet and C.A. Nunez, Aspects of the free field description of string theory on $\operatorname{AdS}(3)$, J. High Energy Phys. 06 (2000) 033.

[6] J. M. Maldacena and H. Ooguri, Strings in $\operatorname{AdS(3)}$ and the SL(2,R) WZW model. Part 3. Correlation functions, Phys. Rev. D 65, 106006 (2002).

[7] G. Giribet and C. A. Nunez, Correlators in $\operatorname{AdS}(3)$ string theory, J. High Energy Phys. 06 (2001) 010.

[8] G. Giribet and C. A. Nunez, Interacting strings on AdS(3), J. High Energy Phys. 11 (1999) 031.

[9] K. Becker and M. Becker, Interactions in the $\operatorname{SL}(2, R) / U(1)$ black hole background, Nucl. Phys. B418, 206 (1994).

[10] J. Teschner, Operator product expansion and factorization in the $H+(3)$ WZNW model, Nucl. Phys. B571, 555 (2000).
[11] J. Teschner, On structure constants and fusion rules in the SL $(2, C) / \mathrm{SU}(2)$ WZNW model, Nucl. Phys. B546, 390 (1999).

[12] J. de Boer, J. Manschot, K. Papadodimas, and E. Verlinde, The Chiral ring of $\operatorname{AdS}(3) / \operatorname{CFT}(2)$ and the attractor mechanism, J. High Energy Phys. 03 (2009) 030.

[13] M. R. Gaberdiel and I. Kirsch, Worldsheet correlators in $\operatorname{AdS}(3) / C F T(2)$, J. High Energy Phys. 04 (2007) 050.

[14] A. Dabholkar and A. Pakman, Exact chiral ring of AdS(3)/CFT(2), Adv. Theor. Math. Phys. 13, 409 (2009).

[15] A. Pakman and A. Sever, Exact $N=4$ correlators of $\operatorname{AdS}(3) / C F T(2)$, Phys. Lett. B 652, 60 (2007).

[16] G. Giribet, A. Pakman, and L. Rastelli, Spectral flow in AdS(3)/CFT(2), J. High Energy Phys. 06 (2008) 013.

[17] C. A. Cardona and C. A. Nunez, Three-point functions in superstring theory on $\operatorname{AdS}(3) \times \mathrm{S}(3) \times \mathrm{T}(4)$, J. High Energy Phys. 06 (2009) 009.

[18] G. E. Giribet and D. E. Lopez-Fogliani, Remarks on free field realization of $S L(2, R)(k) / U(1) \times U(1) \quad$ WZNW model, J. High Energy Phys. 06 (2004) 026.

[19] A. Giveon, D. Kutasov, E. Rabinovici, and A. Sever, Phases of quantum gravity in $\operatorname{AdS}(3)$ and linear dilaton backgrounds, Nucl. Phys. B719, 3 (2005).

[20] M. R. Gaberdiel, R. Gopakumar, and C. Hull, Stringy AdS(3) from the worldsheet, J. High Energy Phys. 07 (2017) 090 . 
[21] K. Ferreira, M. R. Gaberdiel, and J. I. Jottar, Higher spins on $\operatorname{AdS}(3)$ from the worldsheet, J. High Energy Phys. 07 (2017) 131.

[22] G. Giribet, C. Hull, M. Kleban, M. Porrati, and E. Rabinovici, Superstrings on $\operatorname{AdS}(3)$ at $k=1$, J. High Energy Phys. 08 (2018) 204.

[23] M. R. Gaberdiel and R. Gopakumar, Tensionless string spectra on AdS(3), J. High Energy Phys. 05 (2018) 085.

[24] L. Eberhardt, M. R. Gaberdiel, and R. Gopakumar, The Worldsheet Ddual of the symmetric product CFT, J. High Energy Phys. 04 (2019) 103.

[25] L. Eberhardt and M. R. Gaberdiel, String theory on $A d S(3)$ and the symmetric orbifold of Liouville theory, Nucl. Phys. B948, 114774 (2019).

[26] L. Eberhardt and M. R. Gaberdiel, Strings on $\mathrm{AdS}_{3} \times \mathrm{S}^{3} \times \mathrm{S}^{3} \times \mathrm{S}^{1}$, J. High Energy Phys. 06 (2019) 035 .

[27] J. M. Maldacena, H. Ooguri, and J. Son, Strings in AdS(3) and the SL(2,R) WZW model. Part 2. Euclidean black hole, J. Math. Phys. (N.Y.) 42, 2961 (2001).

[28] V. Fateev, A. B. Zamolodchikov, and Al. Zamolodchikov (unpublished).

[29] S. Ribault, Knizhnik-Zamolodchikov equations and spectral flow in $\operatorname{AdS}(3)$ string theory, J. High Energy Phys. 09 (2005) 045.

[30] T. Fukuda and K. Hosomichi, Three point functions in sineLiouville theory, J. High Energy Phys. 09 (2001) 003.

[31] G. Giribet and Y. Nakayama, The Stoyanovsky-RibaultTeschner map and string scattering amplitudes, Int. J. Mod. Phys. A 21, 4003 (2006).
[32] E. Herscovich, P. Minces, and C. Núnez, Winding strings in AdS(3), J. High Energy Phys. 06 (2006) 047.

[33] A. V. Stoyanovsky, A relation between the KnizhnikZamolodchikov and Belavin-Polyakov-Zamolodchikov systems of partial differential equations, arXiv:math-ph/ 0012013.

[34] S. Ribault and J. Teschner, $H+(3)-W Z N W$ correlators from Liouville theory, J. High Energy Phys. 06 (2005) 014.

[35] Y. Hikida and V. Schomerus, $H+(3)$ WZNW model from Liouville field theory, J. High Energy Phys. 10 (2007) 064.

[36] G. Giribet, Violating the string winding number maximally in Anti-de Sitter space, Phys. Rev. D 84, 024045 (2011); Erratum, Phys. Rev. D 96, 024024(E) (2017).

[37] M. Goulian and M. Li, Correlation Functions in Liouville Theory, Phys. Rev. Lett. 66, 2051 (1991).

[38] A. Giveon and D. Kutasov, Notes on $\operatorname{AdS}(3)$, Nucl. Phys. B621, 303 (2002).

[39] P. Baseilhac and V. A. Fateev, Expectation values of local fields for a two-parameter family of integrable models and related perturbed conformal field theories, Nucl. Phys. B532, 567 (1998).

[40] V. A. Fateev and A. V. Litvinov, Multipoint correlation functions in Liouville field theory and minimal Liouville gravity, Theor. Math. Phys. 154, 454 (2008).

[41] A. B. Zamolodchikov and A. B. Zamolodchikov, Structure constants and conformal bootstrap in Liouville field theory, Nucl. Phys. B477, 577 (1996).

[42] G. Giribet, On spectral flow symmetry and KnizhnikZamolodchikov equation, Phys. Lett. B 628, 148 (2005). 